\section{Tolerating enzyme delivery}

\section{By Tim Fulmer, Senior Writer}

Researchers from the University of California, Los Angeles and BioMarin Pharmaceutical Inc. have published in the Journal of Clinical Investigation a strategy for boosting the efficacy of enzyme replacement therapies in lysosomal storage disorders by inducing immune tolerance. Timing and dosing of the tolerance regime will be the major challenges to such an approach.

Lysosomal storage disorders (LSDs) are genetic diseases characterized by absent or malfunctioning lysosomal enzymes that lead to aberrant metabolism of proteins, carbohydrates or lipids in the lysosome. These diseases have varying degrees of severity, but all involve the buildup of enzyme substrate in the lysosome that affects tissue and organ function.

Enzyme replacement therapy (ERT) has proven successful in many of these disorders. However, ERTs can engender an immune response, and high levels of serum antibodies can affect pharmacokinetics, stability and tissue uptake.

Although an antibody response does not have a significant clinical impact in terms of safety or efficacy in most patients on ERT, Susan Richards, VP of immunology R\&D at ERT specialist Genzyme Corp., noted "there is a small subset of patients receiving enzyme replacement therapy where the development of an immune response has been associated with poor clinical outcomes. These are typically enzyme-null patients whose genetic defect results in the inability to produce any enzyme."

The development of an immune response also may depend on the antibody isotype generated. One isotype might affect efficacy by blocking enzyme uptake, while another might affect safety by triggering an inappropriate immune response. Thus, researchers need to determine the effects of antibody isotypes on enzyme uptake and activity, said Whaijen Soo, SVP of R\&D at Shire plc, which also markets ERT products.

The UCLA/BioMarin researchers studied a subset of LSDs, mucopolysaccharide storage disorders. BioMarin and partner Genzyme market Aldurazyme laronidase, a recombinant form of human $\alpha$-L-iduronidase, to treat mucopolysaccharidosis I (MPS I), which is caused by mutations in the gene encoding $\alpha$-L-iduronidase. The therapy delivers properly functioning exogenous enzyme to the lysosome, thus increasing substrate turnover and improving tissue function.

The product's label states that "in clinical studies, 50 of 55 patients (91\%) treated with Aldurazyme were positive for antibodies to laronidase. The clinical significance of antibodies to Aldurazyme is not known, including the potential for product neutralization."

Previously, BioMarin researchers in collaboration with UCLA used
MPS I canine models to develop a strategy for inducing immune tolerance to Aldurazyme. They found that giving Aldurazyme at subtherapeutic doses (about tenfold lower than the therapeutic dose) in combination with a 60-day course of the immunosuppressive drugs cyclosporin A and azathioprine would prevent a host antibody response to subsequent administration of a therapeutic dose of Aldurazyme. ${ }^{1}$

In the JCI paper, the two groups investigated whether the strategy could improve the efficacy of Aldurazyme in the same canine MPS I models. ${ }^{2}$

In MPS I dogs that received previous immune tolerization, i.v. administration of a therapeutic dose of Aldurazyme resulted in enzyme levels that were $260 \%$ higher in lung, heart, kidney and synovial tissues compared with the same tissues in dogs that had not been tolerized. Moreover, increased Aldurazyme correlated with reduced enzyme substrate and decreased tissue pathology compared with non-tolerant controls.

Also, Aldurazyme levels in macrophage-rich tissues such as the liver, spleen and lymph nodes were increased in MPS I dogs that didn't receive the immune tolerization regimen. This suggested that antibodies bound Aldurazyme and redirected it to tissue macrophages, where the drug would be expected to have negligible clinical benefit because the disease's pathology often occurs in skeletal, connective and neurological tissue and not in macrophage-enriched organs.

"The issue with antibodies is real and important," said Gregory Grabowski, professor and director of the Division of Human Genetics at the Cincinnati Children's Hospital Medical Center. "The most important issue is the mechanism of how antibodies diminish the efficacy of administered enzyme."

According to Grabowski, "the obvious effect of antibodies suggested by the JCI paper is that the Fc receptor on macrophage-like cells acts as a sink for antibody-enzyme complexes and thus diverts enzyme away from the target sites of pathology."

This mechanism, he added, particularly applies to storage diseases that are not targeting macrophages, which includes the MPS diseases.

\section{Trials and tribulations}

In the near term, BioMarin will begin testing the immune tolerance strategy in patients with MPS I, said CMO Emil Kakkis, who is corresponding author on the JCI paper.

A study in patients who carry null mutations in the iduronidase gene might be a good place to start, and it could help address whether the JCI immune tolerization strategy is effective against severe forms of MPS I such as Hurler syndrome, said Elizabeth Neufeld, professor of biological chemistry at UCLA. Such patients are unable to express any endogenous enzyme and would thus be expected to mount an antibody response against an ERT such as Aldurazyme, she told SciBX.

Trials also could enroll patients with intermediate or attenuated forms of MPS I-Hurler-Scheie and Scheie syndromes-who express mutant variants of the enzyme with reduced activity or express very small quantities of wild-type enzyme. But Neufeld suggested that studies in patients with severe MPS I would be more fruitful, as patients with the lesser forms apparently are not adversely affected by an immune response 
and eventually can become tolerant to enzyme replacement therapy.

Genzyme's Richards said the ideal immune tolerance strategy "would be one that specifically reduces antibodies to the enzyme replacement therapy, is of short duration, does not induce generalized immunosuppression and can be readily stopped if any clinical issues develop with the patient."

Richards noted the immune tolerance strategy described in JCI required that patients be tolerized before ERT. Thus, she cautioned, it may not be successful in patients who have already begun therapy and mounted an immune response.

Another potential limitation is the requirement for an initial subtherapeutic dose. Although this might work for MPS, it might not be clinically appropriate to delay administering a therapeutic dose in other LSDs, said Richards.

Indeed, dosing studies to determine the length of time it takes to reach immune tolerance would be a key next step, said Shire's Soo. "Some lysosomal storage disorders affect very young children and functional decline is rapid. Waiting for immune tolerance to occur before being able to give a therapeutic dose may be considered unethical," he said.

Shire markets enzyme replacement therapies Elaprase idursulfase and Replagal agalsidase to treat MPS II (Hunter syndrome) and Fabry's disease, respectively.

Richards and colleagues at Genzyme have studied a variety of immune tolerance induction strategies in mouse models of Fabry's disease and Pompe's disease. In both studies, a methotrexate regimen substantially reduced levels of antibody against enzyme replacement therapy compared with other immunosuppressant regimens, including the regimen used in the JCI paper., ${ }^{3,4}$

Genzyme markets three enzyme replacement therapies: Cerezymeimiglucerase, Fabrazyme agalsidase beta and Myozyme alglucosidase alfa, to treat type 1 Gaucher's disease, Fabry's and Pompe's respectively.

The company is evaluating immune tolerance induction regimens in cases "where a defined patient population is at high risk for developing a deleterious antibody response," said Richards.

\section{Model behavior}

Soo argued that the results in canines could use further validation. "As dogs are known to be hypersensitive to antibody production, one needs to be cautious when extrapolating data from such animal studies to potential effects in humans," he said. "If available, other models such as monkey and pig may provide more useful information."

Patricia Dickson, first author on the JCI paper and assistant professor and researcher in the Department of Pediatrics at the Harbor-UCLA Medical Center, told SciBX that her lab is continuing to use the canine MPS I models to test the hypothesis that laronidase therapy begun at birth can induce long-term immune tolerance to the drug. If so, antibodies against the drug might be less of a problem in adulthood.

The UCLA researchers also are following the canine results to investigate ways to deliver ERT more effectively.
Inaccessible compartments such as the brain and the cartilaginous joint space also present a challenge to enzyme replacement therapies in lysosomal storage disorders, and severe forms of many LSDs often have CNS involvement.

Dickson told SciBX she is running a trial of Aldurazyme delivered directly into the spinal fluid in MPS I patients. This strategy "allows direct access of the enzyme to the central nervous system and uses the natural circulation of the spinal fluid to allow wide distribution to brain, spinal cord and meninges." The strategy has shown efficacy in the MPS I canine models, she said.

"Modification of enzymes to allow them to cross the blood-brain barrier into the nervous system from an intravenous injection is also a promising approach," Dickson added.

Meanwhile, Dwight Koeberl, professor in the Division of Medical Genetics and Department of Pediatrics at the Duke University Medical Center, suggested that gene therapy could offer advantages over ERT, including the induction of immune tolerance.

"Gene therapy strategies have advantages including the potential for a curative effect and the stimulation of $\mathrm{T}_{\text {reg }}$ cells to achieve antigen-specific immune tolerance," he said.

In a mouse $\alpha$-glucosidase knockout model of Pompe's disease, Koeberl and colleagues showed that low-dose $\alpha$-glucosidase gene therapy before enzyme replacement therapy prevented subsequent induction of antibodies to the enzyme compared with treatment with a mock gene therapy vector. ${ }^{5}$

Taking another tack, Shire has decided to concentrate on improved enzyme delivery vehicles for targeting tissues and organs, said Soo. "We also believe it is more important to produce a less immunogenic protein than to rely on immunotolerance for improved efficacy," he said.

Kakkis told SciBX that BioMarin has filed multiple patent applications in the U.S., Europe and Japan on methods, use and composition of antigen-specific immune tolerance induction strategies. The company did not disclose further details on the upcoming trial.

In addition to Aldurazyme, BioMarin markets the enzyme replacement therapy Aryplase galsulfase to treat MPS IV.

\section{REFERENCES}

1. Kakkis, E. et al. Proc. Natl. Acad. Sci. USA 101, 829-834 (2004)

2. Dickson, P. et al. J. Clin. Invest.; published online July 24, 2008; doi:10.1172/JCI34676

Contact: Emil Kakkis, BioMarin Pharmaceutical, Novato, Calif. e-mail: ekakkis@bmrn.com

3. Garman, R. et al. Clin. Exp. Immunol. 137, 496-502 (2004)

4. Munroe, J. et al. Clin. Exp. Immunol. 152, 138-146 (2008)

5. Sun, B. et al. Am. J. Hum. Genet. 81, 1042-1049 (2007)

\section{COMPANIES AND INSTITUTIONS MENTIONED}

BioMarin Pharmaceutical Inc. (NASDAQ:BRMN; SWX:BMRN), Novato, Calif.

Cincinnati Children's Hospital Medical Center, Cincinnati, Ohio

Duke University Medical Center, Durham, N.C.

Genzyme Corp. (NASDAQ:GENZ), Cambridge, Mass.

Harbor-UCLA Medical Center, Torrance, Calif.

Shire plc (LSE:SHP; NASDAQ:SHPGY), Basingstoke, U.K. University of California, Los Angeles, Los Angeles, Calif. 